\title{
Das Mammut von Polch bei Mayen (Eifel)
}

\author{
Wighart V. KOENIGSWALD*) \\ Upper Pleistocene, loess, fossil locality, Mammuthus primigenius, skeleton (vertebrae), \\ jaws, teeth (molars), biometry, \\ Rhineland-Palatinate, Rhenish Schiefergebirge, Eifel Mountains.
}

TK25 Nr.: 5609

\begin{abstract}
Kurzfassung: Die Skelettreste des 1936 bei Polch gefundenen Mammutes (Mammutbus primigenius) werden beschrieben. Es handelt sich um ein sehr altes Tier von rund 70 Jahren. Jedoch sind die Epiphysen an den Wirbeln noch nicht vollständig verknöchert. Die unteren $M_{3}$ wirken wesentlich stärker ausgekaut als die zugehörigen oberen $\mathrm{M}^{3}$. Senile Züge zeigt der Unterkiefer aus Polch noch nicht. Diese werden an einem Vergleichstück aus Nierstein/Rhein aufgezeigt.
\end{abstract}

\section{[The Mammoth from Polch near Mayen (Eifel, Western Germany)]}

\begin{abstract}
Skeletal remains of a mammoth (Mammuthus primigenius) were excavated at Polch near Mayen (Eifel) in 1936. The age of this individuum is about 70 years. Despite of this age the epiphyseal disks are not totally fused to the centra of the vertebrae. Lower third molars seem to be much more worn than the upper molars of the same individual. No traces of senility can be seen in the mandibule from Polch. A mandibule with a molar worn out even much more is discribed from Nierstein/Rhine.
\end{abstract}

\section{Einleitung}

Ein Parkplatz an der Autobahn Koblenz - Trier, etwa $12 \mathrm{~km}$ westlich des Koblenzer Kreuzes, hat den ungewöhnlichen Namen „Mammutgrube”. Die topographischen Karten verzeichnen etwa $1 \mathrm{~km}$ westlich davon eine Lokalität „Mammutfundgrube” oder gar „Mammutfanggrube”. Diese Namen erinnern an den Fund von Mammutknochen im Januar des Jahres 1936, die bei Einebnungsarbeiten vom Reichsarbeitsdienst im jungpleistozänen Löß, der hier das devonische Grundgebirge abdeckt, gefunden wurden. Die Konzentration der Knochen auf einen engen Raum ließ vermuten, daß es sich um ein einzelnes Indivi-

*) Anschrift des Autors: Prof. Dr. W. v. Koenigswald, Institut für Paläontologie, Nussallee 8, D-5300 Bonn 1. duum handelt, was sich auch in der Zusammensetzung des Fundgutes bestätigt. Der Fundort liegt am rechten Hang des Nettetales etwa $2 \mathrm{~km}$ nördlich von Polch, bzw. 7 km östlich von Mayen (Blatt 5609 Mayen, R 2594230 H 5576 320).

Die Ausgrabung der Knochen erfolgte im Januar 1936 unter der Leitung des Geologen Dr. J. NIEDERMAYER vom Geologisch-Paläontologischen Institut der Universität Bonn, von dem lediglich Zeitungsberichte über den Fund vorliegen (NIEDERMAYER 1936a und b), weil er bald nach der Ausgrabung Bonn verließ.

Der Fund führte zu einem heftigen Disput. Während Niedermayer (1936a und b) und MülleR-DeILE (1939) in den fossilen Knochen die Reste eines auf natürliche Weise zu Tode gekommenen Tieres sahen, konnten sich MORDZIOL (1936, 1939) und MAUZ (1939) das Mammut nur als Jagdbeute des frühen Menschen vorstellen. Diese für sie ganz offensichtliche Tatsache versuchten sie noch nach der Grabung zu belegen. Als Argumente dienten ihnen u. a. der Umstand, daß die Skelett-Teile in einer kleinen Depression in der Oberfläche des unterlagernden Devons gefunden wurden. Darin sahen sie eine vom Menschen gegrabene „Fallgrube” oder zumindest eine "Stolpergrube". Ein Wildwechsel wurde rekonstruiert, der einer kleinen Abflußrinne entsprochen haben soll und schließlich wurden Quarzstücke als Artefakte interpretiert.

Wer jemals im arktischen Permafrostboden Grabungen durchgeführt hat oder auch nur hierzulande versucht hat, einen tief gefrorenen Boden aufzugraben, wird berechtigten $Z$ weifel daran haben, daß es für den Jäger des Jungpaläolithikums lohnend gewesen sein dürfte, im Permafrostboden wirkungsvolle Fallgruben anzulegen. Nachuntersuchungen durch HoFER (1939) zeigten, daß kleine Depressionen in der Devon-Oberfläche verbreitet sind und deswegen nicht als künstliche Anlage des Menschen angesehen werden können. Die angeblichen Artefakte müssen 
als lokal ausgewitterte Gangquarze angesprochen werden (BOSINSKI 1979). Damit bleibt kein stichhaltiges Argument, um das Mammut als Jagdbeute zu interpretieren und erst recht fehlt jeder Hinweis auf eine "Fallgrube” an einem Wildwechsel. Wenn man bedenkt, daß das Mammut mit über 60 Jahren zur höchsten Altersgruppe gehörte (Kap. 4.3), dann ist ein natürlicher Tod nicht unwahrscheinlich.

Über die widersprüchlichen Ansichten, ob Jagdplatz oder nicht, wurde von MAUZ (1939), MORDZIOL
(1939), MÜlLER-DeILE(1939) und HOFER (1939) zwar ausführlich berichtet, aber die paläontologischen Angaben über den Mammutfund selber gerieten äußerst spärlich. Sie sollen hier, so weit wie möglich, nachgeholt werden.

\section{Der Umfang des Skelettes}

Die Knochen des "Polcher Mammuts” kamen an das Geol.-Paläontol. Institut der Universität Bonn und befinden sich noch heute in der Sammlung des

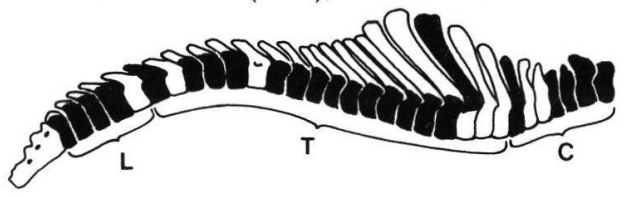

B

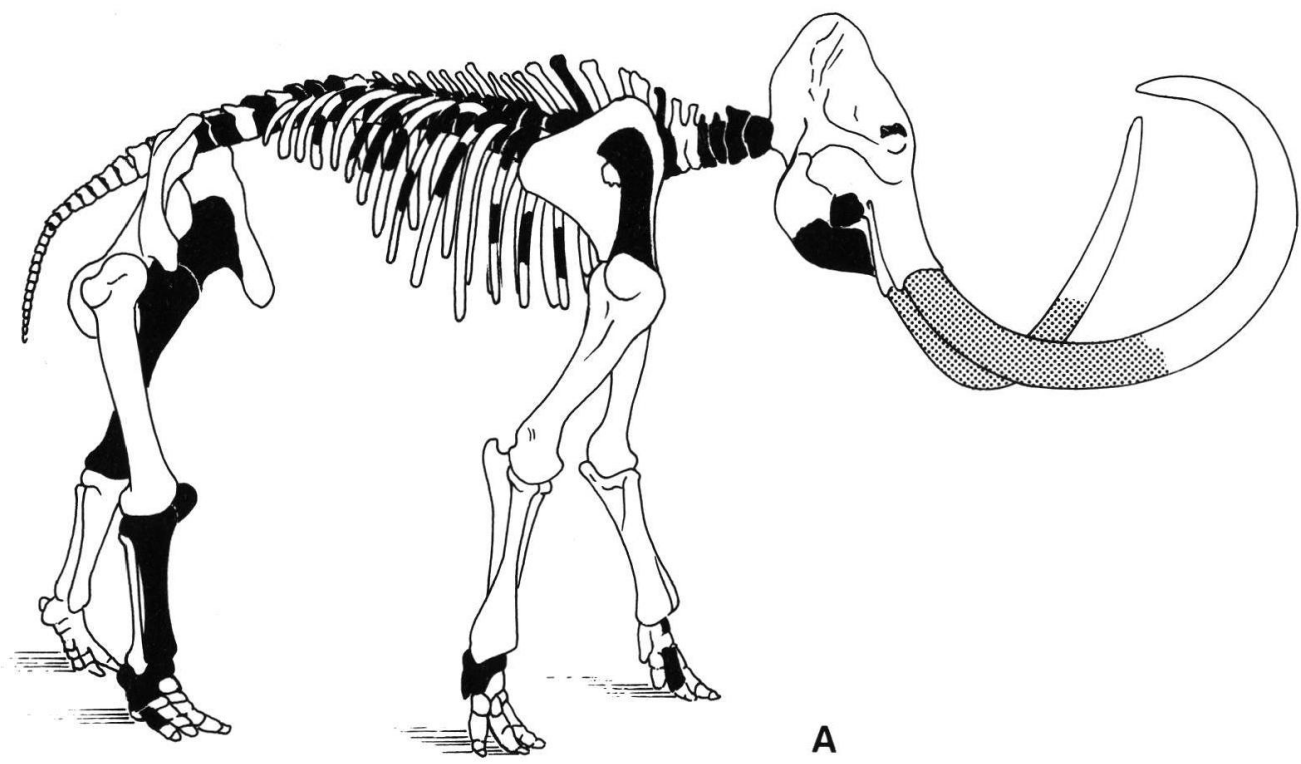

C

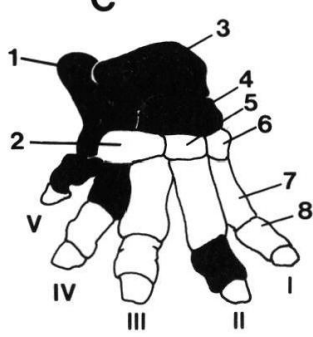

D

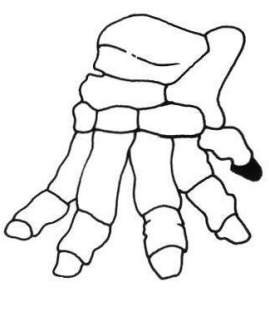

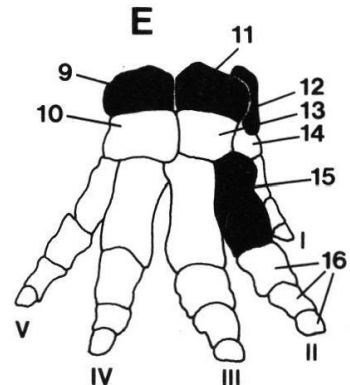

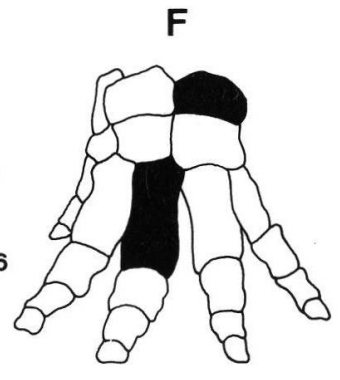

Abb. 1: Mammut von Polch. A In die Zeichnung des Mammutskelettes aus Borna bei Leipzig (nach ABEL 1929) wurden die erhaltenen Teile des Mammutes von Polch schwarz eingetragen. Die nicht mehr überlieferten Stoßzähne wurden nach einem Foto von MAUZ (1939) gerastert eingetragen. B Wirbelsäule vom Mammut mit Eintragung der erhaltenen Wirbel $\mathrm{C}=$ Cervicalwirbel, $\mathrm{T}=$ Thoracalwirbel und $\mathrm{L}=$ Lumbalwirbel (nach TOEPFER 1957). $\mathrm{C}$ rechter Hinterfuß: $1=$ Calcaneus, 2 = Cuboid, $3=$ Astragalus, $4=$ Naviculare, $5=$ Tarsale II, $6=$ Tarsale I, $7=$ Metatarsalia, $8=$ Phalangen; D linker Hinterfuß; E rechter Vorderfuß: $9=$ Ulnare, $10=$ Carpale IV-V, $11=$ Intermedium, $12=$ Radiale, $13=$ Carpale III, 14 = Carpale II, 15 = Metacarpalia, 16 = Phalangen; F linker Vorderfuß (C bis F nach OLsON 1972). 


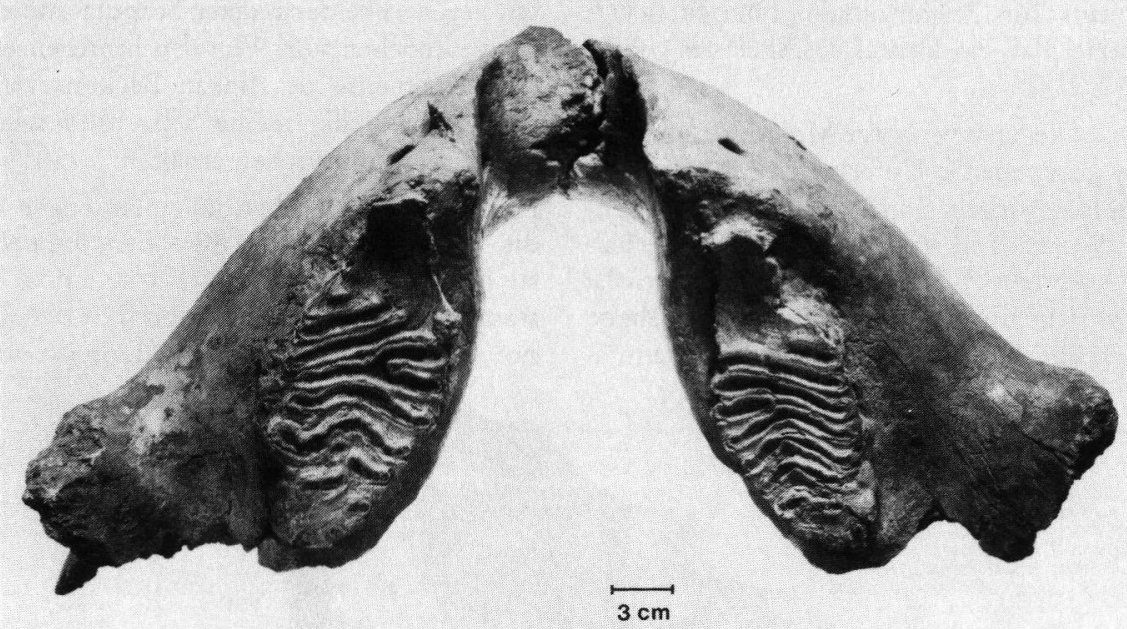

Abb. 2: Mammut von Polch. - Unterkiefer mit den beiden stark ausgekauten $M_{3}$. Das Tier gehört zur höchsten Altersgruppe und dürfte etwa 70 Jahre alt geworden sein.
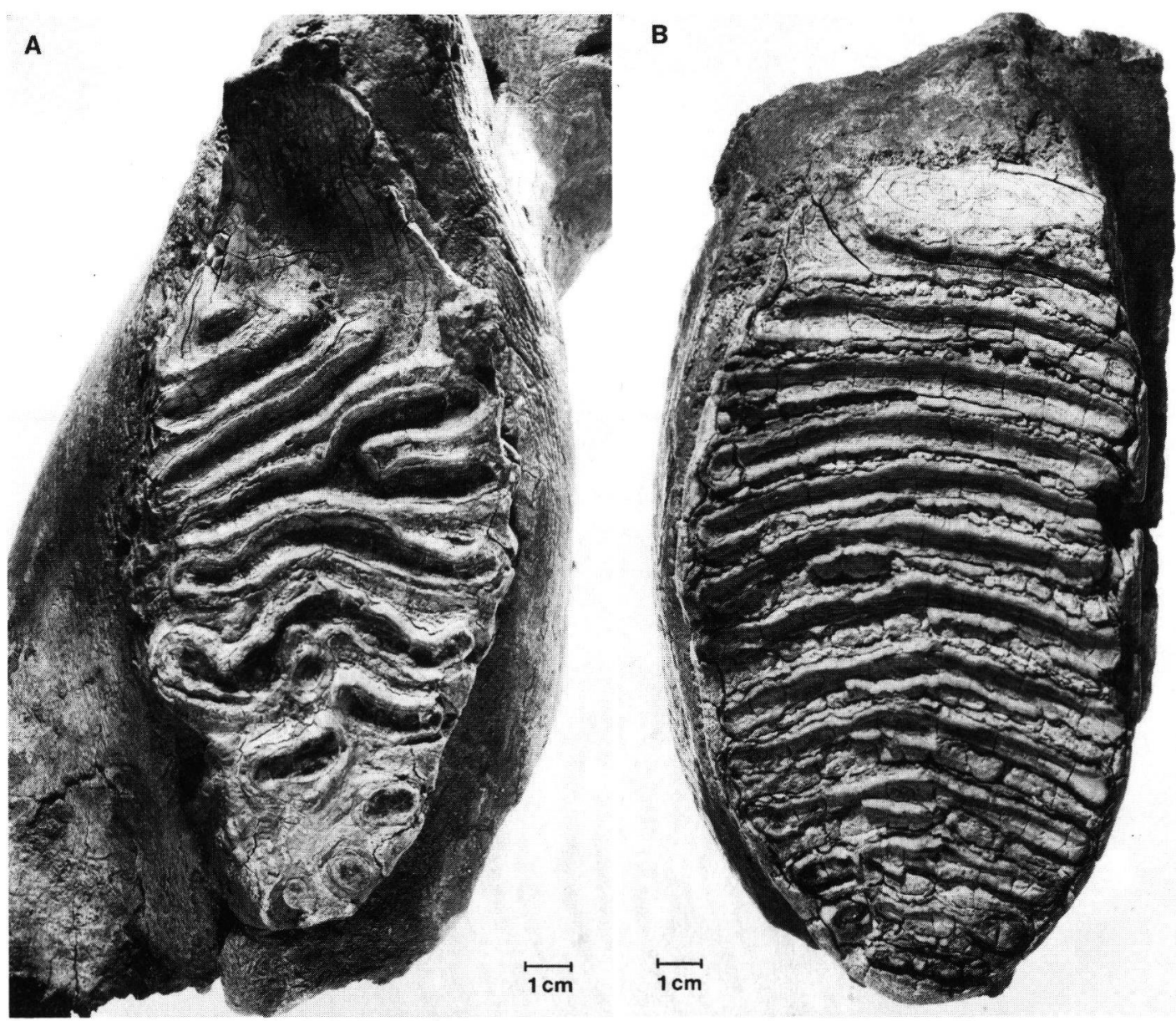

Abb. 3: Mammut von Polch. - A Linker $\mathrm{M}_{3}$ des Unterkiefers und $\mathbf{B}$ rechter $\mathrm{M}^{3}$ des Oberkiefers. In dieser Altersgruppe ist der untere $M_{3}$ stärker abgekaut als der obere $\mathrm{M}^{3}$, wie diese von einem Individuum stammenden Zähne zeigen. 
Bonner Instituts für Paläontologie. NIEDERMAYER (1936b) schätzte, daß ein Drittel des Skelettes geborgen wurde.

An Gebißresten liegen zwei obere Molaren und ein in der Mediane gebrochener Unterkiefer mit zwei $\mathrm{M}_{3}$ vor. Von den Halswirbeln sind 5, von Brust- und Lendenwirbeln 19 zum Teil stark fragmentarisch erhalten. Hinzu kommen 3 Schwanzwirbel. Zahlreiche Rippenbruchstücke und das recht selten beschriebene Brustbein sind überliefert. Von den Vorderextremitä- ten liegen Teile der rechten Scapula und einige Handwurzelknochen vor. Von den hinteren Extremitäten sind Fragmente des linken Beckens und das linke Femur sowie die rechte Tibia und wiederum eine Reihe der Fußknochen erhalten.

Der Fund der Knochen auf einem engen Raum sowie die Tatsache, daß kein Knochen doppelt belegt ist, stellt sicher, daß alle Reste von einem Individuum stammen, wie es bereits von NIEDERMAYER angenommen wurde. Die Feststellung ist wichtig, weil
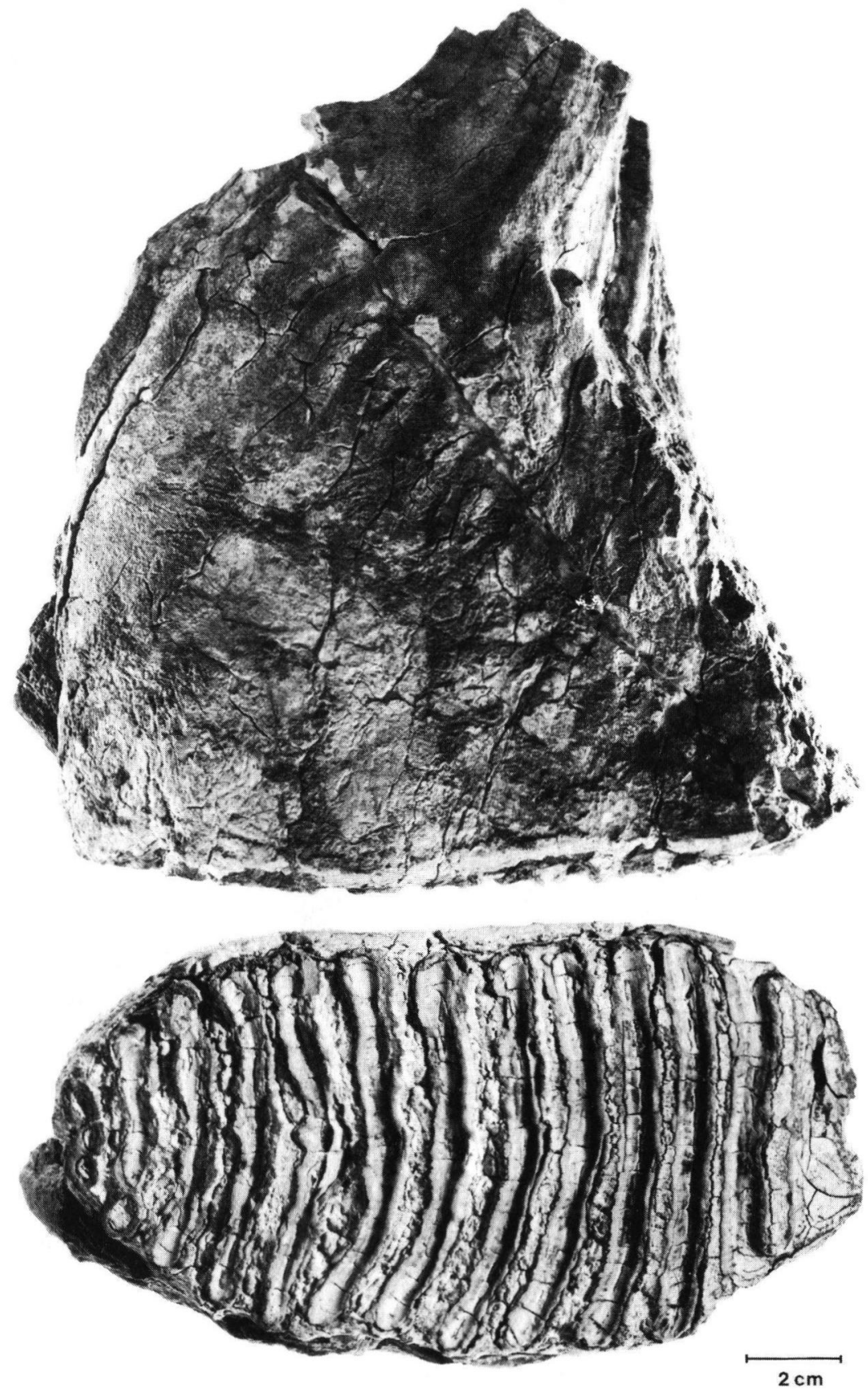

Abb. 4: Mammut von Polch. - Der linke $\mathrm{M}^{3}$ besitzt noch eine maximale Kronenhöhe von $15 \mathrm{~cm}$. 
nur auf dieser Basis sinnvolle Angaben über das individuelle Alter und den Vergleich des Abkauungszustandes der Molaren sowie die Schließung der Epiphysenfugen möglich sind.

Es gibt keine Fundpläne oder Fundverzeichnisse (? mehr). MAUZ (1939) führt auch nur die wichtigsten Knochen auf. Deswegen kann nicht mehr überprüft werden, ob die überlieferten Knochen vollständig sind, oder ob Knochen in der Zwischenzeit verlorengegangen sind. Bei MORDZIOL (1939) und MAUZ (1939) sind einige Fotos der Grabung wiedergegeben, auf denen ein Teil der Knochen zu identifizieren sind. Nach der Liste und den Fotos ergibt sich der Eindruck, daß das Knochenmaterial im wesentlichen vollständig erhalten ist. Nicht mehr nachzuweisen sind aber offensichtlich die beiden Stoßzahnfragmente, die auf dem Foto bei MAUZ (1939: Taf. 1: $a$ und b) zu sehen sind. In Abb. 1 wird der Umfang des vorliegenden Materials graphisch dargestellt.

\section{Beschreibung}

\subsection{Das Gebiß}

Im Unterkiefer, dessen Coronidfortsätze fehlen und dessen Bruch in der Mediale alt sein dürfte, sind die zwei $\mathrm{M}_{3}$ in einem tief abgekauten Zustand erhalten (Abb. 2 und 3). Beide Molaren haben im vorderen Teil, der etwa $6 \mathrm{~cm}$ lang ist, eine glatte Dentinfläche. Die Schmelzlamellen sind in diesem Bereich völlig ausgekaut. Im hinteren Teil sind etwa 6 bis 7 recht unregelmäßige Lamellen zu erkennen, denen ein Talonid mit 3 Schmelzsäulchen folgt. Die Breite der Zähne beträgt rechts maximal 78 und links $88 \mathrm{~mm}$ und dürfte damit bereits stark reduziert sein. Die Höhe der hinteren Schmelzlamellen ist im Kiefer verborgen, muß aber zwischen 2 und $4 \mathrm{~cm}$ liegen. Die Zähne werden als dritte Molaren angesprochen, weil kein nachfolgender Zahn mehr im Kiefer steht. Ohne den Druck eines Nachfolgers sind die $\mathrm{M}_{3}$ auch nicht mehr nach vorne gerückt.

Gegenüber den beiden stark ausgekauten Molaren des Unterkiefers wirken die beiden oberen $\mathrm{M}^{3}$ noch relativ groß, weil die Höhe des Zahnes über den letzten Schmelzlamellen ohne Wurzeln immer noch $15 \mathrm{~cm}$ beträgt (Abb. 3 und 4). Stammten die vier Zähne nicht aus einem geschlossenen Fundverband, in dem nur ein Individuum belegt ist, könnte man verleitet sein, die oberen Molaren einer anderen Abkaustufe zuzuordnen. Es handelt sich eindeutig um $\mathrm{M}^{3}$, weil an beiden Molaren Druckmarken eines nachfolgenden Zahnes fehlen. Darüber hinaus ist am distalen Ende des rechten Zahnes ein Kieferrest erhalten, der ausschließt, daß ein weiterer Zahn gefolgt ist. Die Lamellenformel lautet für beide Zähne oo-11-x. Am mesialen Rand ist eine kurze Dentinplatte erhalten, der 11 nahezu gestreckte Lamellen folgen. Ein Talon

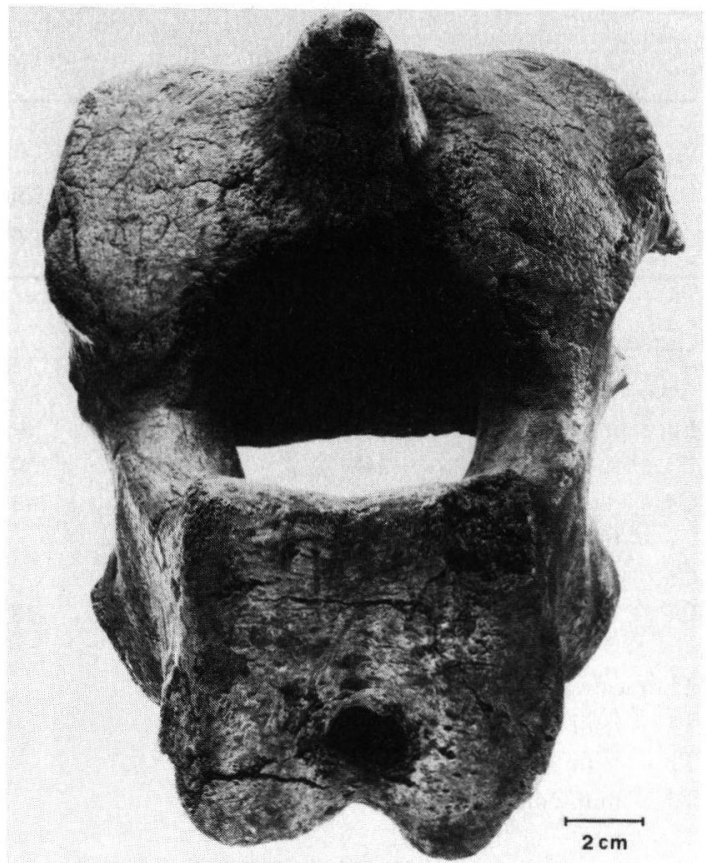

Abb. 5: Mammut von Polch. - Epistropheus mit einem tiefen Loch in der dorsalen Rinne des Processus spinosus.

wird von 3 Schmelzsäulchen gebildet. Die maximale Breite der Zähne (93 mm rechts und $95 \mathrm{~mm}$ links) scheint im Gegensatz zu den unteren Molaren nicht reduziert zu sein.

Auf $10 \mathrm{~cm}$ der Kaufläche kommen 8 Lamellen, womit es sich mit Sicherheit um die jungpleistozäne Art Mammuthus primigenius (BLUMENBACH 1799) handelt. Das paßt zu den Angaben von NIEDERMAYER (1936a) und MORDZIOL (1936), daß der Löß, in dem die Knochen lagen, als jüngerer Löß, also der des letzten Glazials, anzusprechen ist. Das individuelle Alter des Tieres wird aufgrund des Abkauzustandes der Zähne in Kap. 4.3. diskutiert.

\subsection{Die Wirbelsäule und Sternum}

Es sind 27 Wirbel überliefert, deren Maße in Tab. 1 aufgelistet sind. Von den Cervicalwirbeln liegen Atlas und Epistropheus (Abb. 5), sowie drei weitere Halswirbel vor, die als C3, C4 und C7 angesprochen werden. Die Halswirbel haben flache scheibenförmige Wirbelkörper und die Höhe der Dornfortsätze nimmt von cranial nach caudal zu. Der C7 ist an der caudal gelegenen Gelenkfacette für die erste Rippe zu erkennen. Beim vorliegenden Material sind die Fortsätze weitgehend unvollständig.

Die Zahl der Thoracal- und Lumbalwirbel variiert etwas (TOEPFER 1957). Da hier nicht die Gesamtzahl 
Tab. 1: Mammut von Polch, Abmessungen der Wirbel in mm $(-=$ nicht meßbar, $0=$ geschätzt, $*=$ ohne Epiphysenscheiben $)$

Wirbel,

Wirbelkörper

Breite cran.
Höhe cran.
Foramen

Breite vertebrale

Höhe

caud.

caud.

\section{Länge}

Cervicalwirbel

Atlas

Epistropheus

C3

$\mathrm{C} 4$

C5 - fehlt -

C6 - fehlt -

C7

164

178

148

152

140
156

95

110

$\begin{array}{ll}- & 179 \\ 148 & 176\end{array}$

-

148

66

64

-

56

96

52

Thoracalwirbel

T1 - fehlt -

T2 - fehlt -

T3 - nur Dornfortsatz -

T4

T5

T6

T7

T8

T9

T10

T11

T12

$\mathrm{T} 13$

T14 - fehlt -

T15

T16

T17

T18 - fehlt -

T19

$138^{*}$

$\begin{array}{lc}- & 150^{*} \\ - & - \\ - & - \\ - & -\end{array}$

$-$

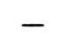

68

67

66

63

73

131

$-$

$127^{*}$

$-$

$-$

123

126

128

$$
-
$$

62

62

60

$-$

$137 *$

$127^{*}$

$134^{*}$

56

52

55

54

Lumbalwirbel

L1 - fehlt -

L2

L3

$134 *$

$143^{*}$

-

-

141*

$-$

84

46

155

$-$

139

$133^{*}$

(129)

$125^{*}$

$-$

89

33

L5

187

$184^{*}$

81

104

104

29

26

Sacrum fehlt

Caudalwirbel

proximaler Bereich

a

b

mittlerer Bereich

c

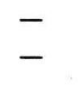

48

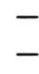

46

42

41 


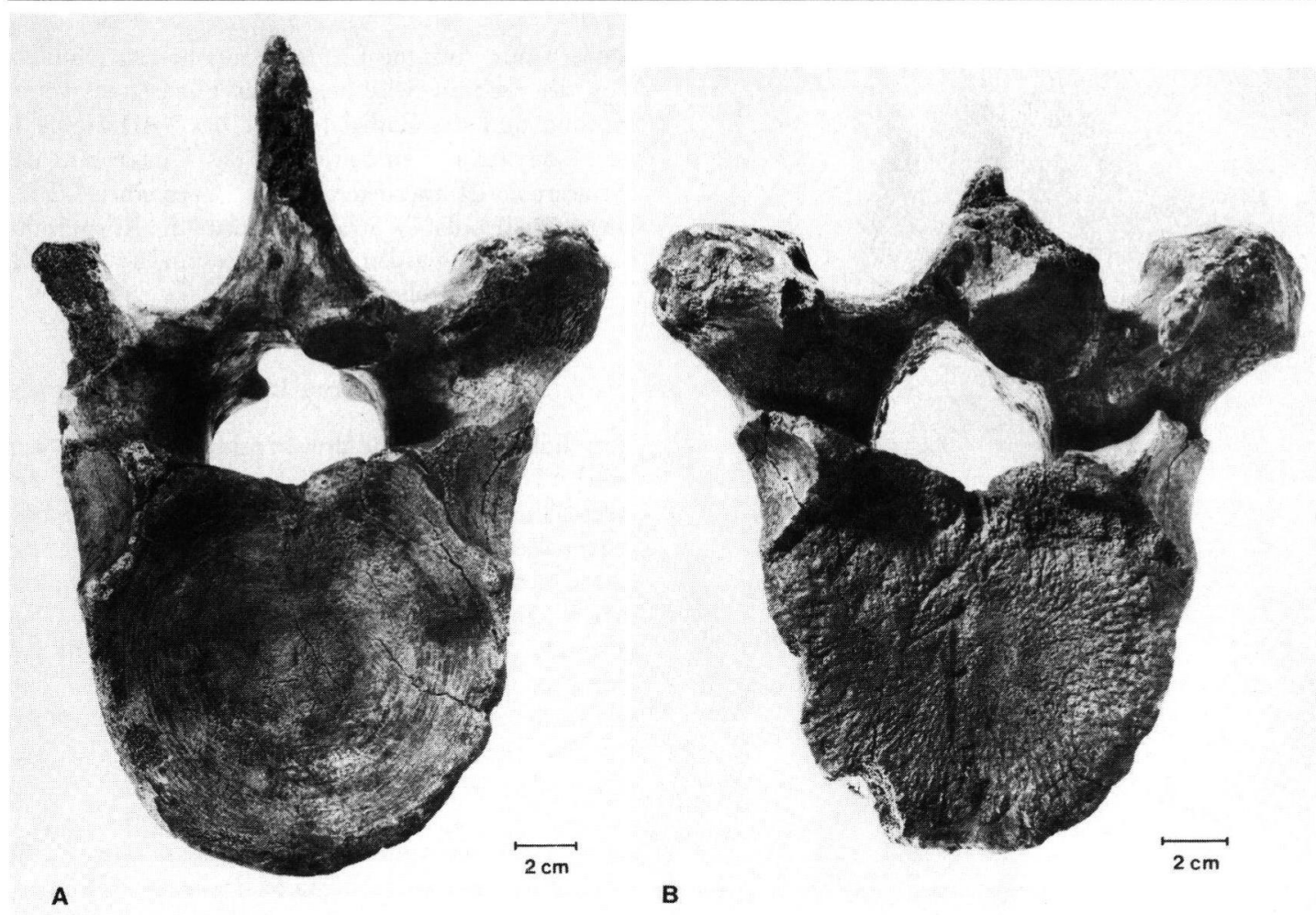

Abb. 6: Mammut von Polch. - 12. und 13. Brustwirbel von caudal mit asymmetrisch ausgebildeten Postzygapophysen. Bei dem 13. Brustwirbel hat sich die nur unvollständig verwachsene Epiphysenscheibe wieder vom Wirbelkörper gelöst.

überprüft werden kann, wird von 19 Thoracal- und 5 Lumbalwirbeln ausgegangen. Von den Thoracalwirbeln sind 15 teils durch Fragmente belegt. Besonders stark sind die Wirbelkörper aus dem vorderen Bereich verwittert. Die Processi spinosi sind in der Regel abgebrochen, so daß ihre Größe, die zunächst bis zum T3 ansteigt und dann kontinuierlich wieder absinkt, nicht zur Bestimmung herangezogen werden. Der Proc. transversus steht im cranialen Thoracalbereich nahezu waagerecht und weist ab dem T13 deutlich schräg nach oben. Die letzten drei Thoracalwirbel (T17, T18 und T19) haben im Gegensatz zu den vorderen nur noch eine Gelenkfacette für die Rippen. Sie liegt etwa in der Mitte des Wirbelkörpers (SIEGFRIED 1983). Nach diesen Merkmalen, sowie nach geringen Asymmetrien konnten die vorhandenen Wirbel eingeordnet werden. Es fehlen aus der Thoracalregion der T1 und T2 sowie T14 und T18. Vom T3 ist nur der Processus spinosus (Länge 435 $\mathrm{mm}$ ) erhalten.

Die Prae- und Postzygapophysen mancher Thoracalwirbel sind etwas asymmetrisch ausgebildet, am T12 und T13 fehlen sie auf der linken Seite (Abb. 6). Obwohl das Mammut von Polch bereits ein hohes individuelles Alter erreicht hat (Kap. 4.3.), sind die Epi- physenscheiben meist nur randlich mit dem Wirbelkörper verknöchert, so daß sie sich häufig wieder gelöst haben (Abb. 6).

Aus der Lumbalregion fehlt nur der L1. Bei den Lumbalwirbeln, die an der fehlenden Facette für die Rippen zu erkennen sind, werden von cranial nach caudal die Wirbelkörper ebenso wie der Neuralkanal kontinuierlich breiter aber niedriger. Das Sacrum ist nicht erhalten.

Von den 21 Caudalwirbeln sind nur drei überliefert. Zwei davon gehören der proximalen Region, in der noch ein Neuralkanal vorhanden ist, und einer dem mittleren Bereich des Schwanzes an.

Aus dem Brustkorb sind 24 größere Rippenfragmente und der caudale Teil des Sternums erhalten. An den meisten aufgestellten Mammutskeletten ist kein Sternum zu sehen, weil die distalen, knorpeligen Rippenabschnitte fehlen. In den Beschreibungen wird das Sternum selten erwähnt, weswegen es hier ausführlicher behandelt werden soll. TOEPFER (1957) beschreibt vom Mammut aus Pfännerhall nur den vorderen Teil des Sternums. Aus Polch liegen der mittlere und hintere Teil synossifiziert vor (Abb. 7), während der vordere fehlt. Der Knochen hat eine Länge von etwa $32 \mathrm{~cm}$ und ist auf der Innenseite glatt, auf 


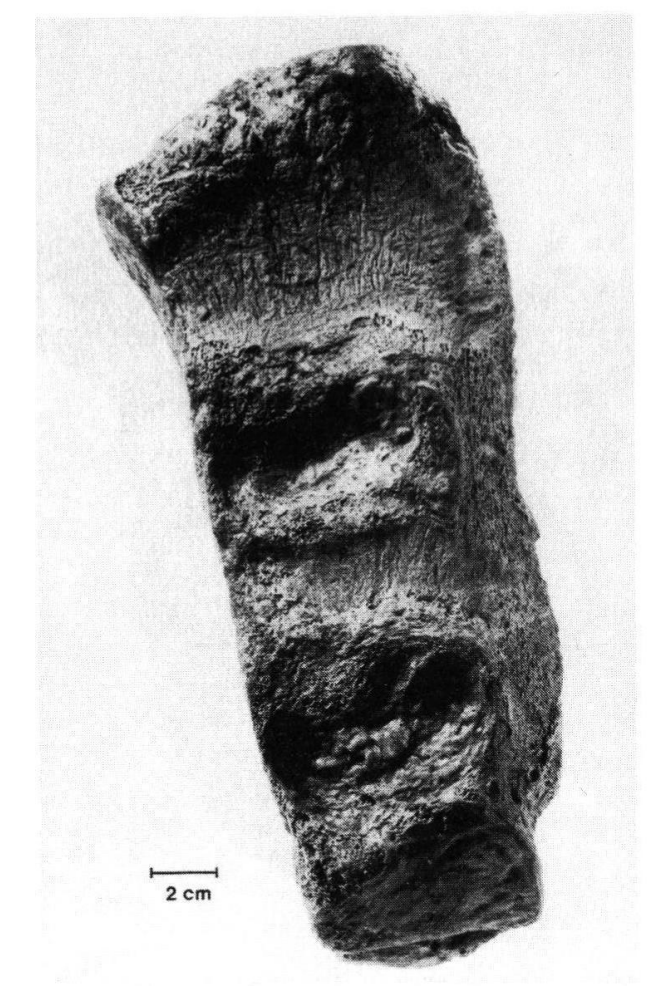

Abb. 7: Mammut von Polch. -

Sternum, rechte Lateralseite des caudalen Sternumabschnittes mit 4 Marken für den Ansatz der knorpeligen Rippen.

der Außenseite gekielt. Auf jeder Seite dieses sehr tiefen Knochens (vorne $12 \mathrm{~cm}$, hinten $8 \mathrm{~cm}$ ) sind vier Ansatzstellen für die knorpeligen Rippenbögen zu erkennen. Die vorderste Marke gehört nach dem Vergleich mit Elephas maximus zum distalen Teil der zweiten Rippe. Ihr anderer Teil sitzt auf dem vordersten Sternum-Teil. Deutlich abgesetzt ist die Marke der dritten Rippe. Es folgen beim Mammut von Polch zwei weitere Marken, während bei dem im Museum Senckenberg aufgestellten Skelett von Elephas maximus noch drei folgen. Dort gehören sie zum 5., 6. und dem gemeinsamen Ende der 7. und 8. Rippe. Für das Mammut von Polch ist denkbar, daß die 5. und 6. Rippe ebenfalls gemeinsam enden oder, daß die letzte Marke den drei letzten Rippen zuzuordnen ist. Es läßt sich aber zur Zeit nicht absehen, wie groß die Variabilität in diesem Merkmal ist.

\subsection{Die vorderen Extremitäten}

Von der rechten Scapula liegen zwei große axiale Fragmente vor, deren Bruch alt ist. Die Gelenkfacette der Scapula mißt 14 × $21 \mathrm{~cm}$. Von den vorderen Extremitäten sind keine Langknochen erhalten, sondern nur einzelne Elemente aus beiden Händen. Von der rechten Seite liegen das Ulnare, das Intermedium und das Radiale, sowie das Metacarpale II vor. Von der linken Seite sind das Ulnare und das Metacarpale III überliefert (Abb. 1). An einer 1. Phalange des III oder IV Strahles konnte die Körperseite nicht bestimmt werden. Die Abmessungen werden in Tab. 2 und 3 gegeben.

\subsection{Die hinteren Extremitäten}

Vom linken Pelvis sind drei Fragmente erhalten, der äußere Teil der Iliumschaufel, der craniale Teil des Acetabulum und ein Stück des Pubis. Die Stücke passen an alten Bruchkanten zusammen. Von den Langknochen ist lediglich das linke Femur mit einer maximalen Länge von $125 \mathrm{~cm}$ erhalten. Das Caput hat einen Durchmesser von $19 \mathrm{~cm}$, das distale Gelenk hat eine max. Breite von 24 und eine Tiefe von $27 \mathrm{~cm}$. Die vorliegende Tibia gehört zur rechten Körperseite. Ihre max. Länge beträgt $69 \mathrm{~cm}$; Breite und Tiefe der proximalen Gelenkung messen 26,5 und $22 \mathrm{~cm}$, am distalen Gelenk 20 und $16 \mathrm{~cm}$. An beiden Knochen sind die Epiphysenfugen völlig geschlossen. Beide Patellae liegen vor (Höhe 15,5; Breite $13 \mathrm{~cm}$ ).

Von den Fußwurzel-, Mittelfußknochen und Phalangen hat sich von der linken Seite nur die 2. Phalange des V. Strahles erhalten. Vom rechten Fuß sind im Anschluß an die rechte Tibia Astragalus, Calcaneus, Naviculare, die Metatarsalia IV und V sowie die 1. Phalange der II. Strahles vorhanden. Die Abmessungen werden in Tab. 2 und 3 aufgelistet.

\section{Ausdeutung}

\subsection{Die Größe des Tieres}

Zur Abschätzung der Größe des Tieres kann am besten die Länge des Femurs und der Tibia herangezogen werden. Sie stimmt recht gut mit dem Mammutskelett von Ahlen (SIEGFRIED 1959) überein, das im Paläontologischen Museum der Universität Münster mit einer Schulterhöhe von 3,20 m und einer Länge von $5,40 \mathrm{~m}$ rekonstruiert ist. Das Mammut von Pfännerhall (ToEPFER 1957) besitzt ein um wenige $\mathrm{cm}$ kürzeres Femur $(122 \mathrm{~cm})$. Der höchste Dornfortsatz der Wirbelsäule wird auch mit $3,20 \mathrm{~m}$ angegeben. Die tatsächliche Schulterhöhe dürfte mit Fettbuckel und Fell noch etwas größer gewesen sein. Die Länge von den Stoßzähnen bis zum Schwanz beträgt 4,60 m. Fast die gleichen Abmessungen dürften für das Mammut von Polch gelten.

Angaben zum Geschlecht des Tieres sind kaum möglich. Nach dem Foto der Stoßzähne waren diese relativ dick und stark gekrümmt, wie es eher für männ- 
Tab. 2: Mammut von Polch. Abmessungen der Metacarpalia, Metatarsalia und Phalangen (in mm):

\begin{tabular}{lccccc}
\hline & Ges. länge & $\begin{array}{c}\text { prox. } \\
\text { Breite }\end{array}$ & $\begin{array}{c}\text { Gelenkende } \\
\text { Tiefe }\end{array}$ & $\begin{array}{c}\text { dist. } \\
\text { Breite }\end{array}$ & $\begin{array}{c}\text { Gelenkende } \\
\text { Tiefe }\end{array}$ \\
\hline Mt IV dex. & 132 & 76 & 88 & 66 & 79 \\
Mt V dex. & 97 & - & 90 & - & 85 \\
1. Phal. II dex. & 80 & 56 & 60 & 49 & 40 \\
2. Phal. V sin. & 60 & 53 & 41 & 47 & 35 \\
Mc II dex. & 188 & 84 & 111 & 81 & 96 \\
Mc III sin. & 192 & 90 & 122 & 86 & 97 \\
1. Phal. III o. IV & 102 & 82 & 79 & 69 & 58 \\
\hline
\end{tabular}

Tab. 3: Mammut von Polch. Abmessungen des Carpus und Tarsus (in $\mathrm{mm}$ ):

\begin{tabular}{|c|c|c|c|c|}
\hline & Gesamtbreite & Gesamttiefe & Gesamtlänge & Dicke \\
\hline Astragalus dex. & 174 & 154 & & 103 \\
\hline Calcaneus dex. & - & 201 & & 142 \\
\hline Naviculare dex. & 154 & & 113 & 42 \\
\hline Ulnare sin. & 150 & 163 & & 76 \\
\hline Ulnare dex. & 157 & 160 & & 73 \\
\hline Intermed. dex. & 142 & 136 & & 83 \\
\hline \multirow[t]{3}{*}{ Radiale dex. } & 103 & & 159 & 65 \\
\hline & \multicolumn{2}{|c|}{$\begin{array}{c}\text { Calcaneus-/ } \\
\text { Astragalusfacetten }\end{array}$} & \multicolumn{2}{|c|}{ Navicularfacette } \\
\hline & Breite & Tiefe & Breite & Länge \\
\hline Astragalus dex. & 156 & 110 & 143 & 85 \\
\hline Calcaneus dex. & 156 & 110 & - & - \\
\hline
\end{tabular}

liche Tiere zu erwarten wäre. Da das Tier aber bereits ein sehr hohes individuelles Alter hatte, ist es sehr fraglich, ob darauf eine zuverlässige Geschlechtszuordnung begründet werden kann.

\subsection{Pathologische Veränderungen}

Der Dornfortsatz des Epistropheus weist ein tiefes rundes Loch auf (Abb. 5), das möglicherweise von einer Fistel stammt. Wie bei vielen Mammutskeletten lassen sich auch am Polcher Mammut leichte Unregelmäßigkeiten am Skelett nachweisen. Einige Thorakalwirbel sind leicht asymmetrisch (Abb. 6). Derartige Asymmetrien sind bei Mammutresten häufig zu beobachten, z. B. in den Weinberghöhlen bei Mauern (KOENIGSWALD 1974). TREVISAN (1948: Abb. 12) bildet wesentlich schwerwiegendere asymmetrische Veränderungen an Wirbeln von Elephas (Palaeoloxodon) antiquus ab. Aus solchen Asymmetrien dürften kaum Behinderungen für diese großen Tiere entstanden sein.

\subsection{Das individuelle Alter}

Der Eindruck der Ausgräber hat sich bestätigt, daß alle Knochen zu einem Individuum gehören. Kein Knochen ist doppelt vorhanden und der Stand des Zahnwechsels paßt zur Verknöcherung der Epiphysenfugen in den Extremitäten. Das Alter läßt sich am besten am Gebiß ablesen. Die letzten Molaren des Unterkiefers sind tief abgekaut und zeigen in ihrem hinteren Teil kein vollständiges Lamellenbild mehr. Der vordere Zahnabschnitt ist bereits ausgekaut und auf die Breite der Wurzel reduziert.

Nach einer von GUENTHER (1955) gegebenen Tabelle dürfte es sich bei diesem Grad der Abkauung um die Altersgruppe von 60-70 Jahren handeln. Für das Mammut von Pfännerhall wird ein Alter von knapp 60 Jahren angegeben (TOEPFER 1957), aber dort ist der untere $M_{3}$ noch weit weniger abgekaut. Deswegen muß das Alter des Mammutes von Polch bei etwa 70 Jahren liegen. Legt man die Werte für Elephas 
maximus zugrunde (ROTH \& SHOSHANI 1988), dann kommt man eher zu noch höheren Alterszahlen.

Auffallend ist der starke Unterschied in der Abkauung zwischen den unteren und oberen $\mathrm{M}^{3}$. Weil die individuellen Unterschiede in der Abkauung in der höchsten Altersstufe besonders groß sind, kann das Abkaubild des Mammuts von Polch nicht ohne weitere Vergleiche verallgemeinert werden. Es erweist sich aber als sehr schwierig, genügend Vergleichsmaterial für Mammute oder Elefanten in dieser Altersgruppe zusammen zu tragen.

Da beim Mammut von Polch die rechten und linken Zahnreihen in gleicher Weise abgekaut sind und die
Kauflächen auch keine untypischen Auskalkungen aufweisen, dürfte kein pathologisch bedingter Sonderfall vorliegen.

Wichtig ist festzuhalten, daß selbst in dieser Altersstufe die Epiphysenscheiben der Wirbel unvollständig mit dem Zentrum verschmolzen sind (Abb. 6). Zumindest in der Diagenese haben sie sich zum Teil wieder gelöst. Aus offenen Epiphysenfugen an den Wirbeln darf man bei Mammuten demnach keineswegs auf ein subadultes Alter schließen.

Da das Mammut von Polch zur höchsten Altersgruppe, die bei Mammuten zu erwarten ist, gehört, ist ein natürlicher Alterstod für dieses Tier durchaus
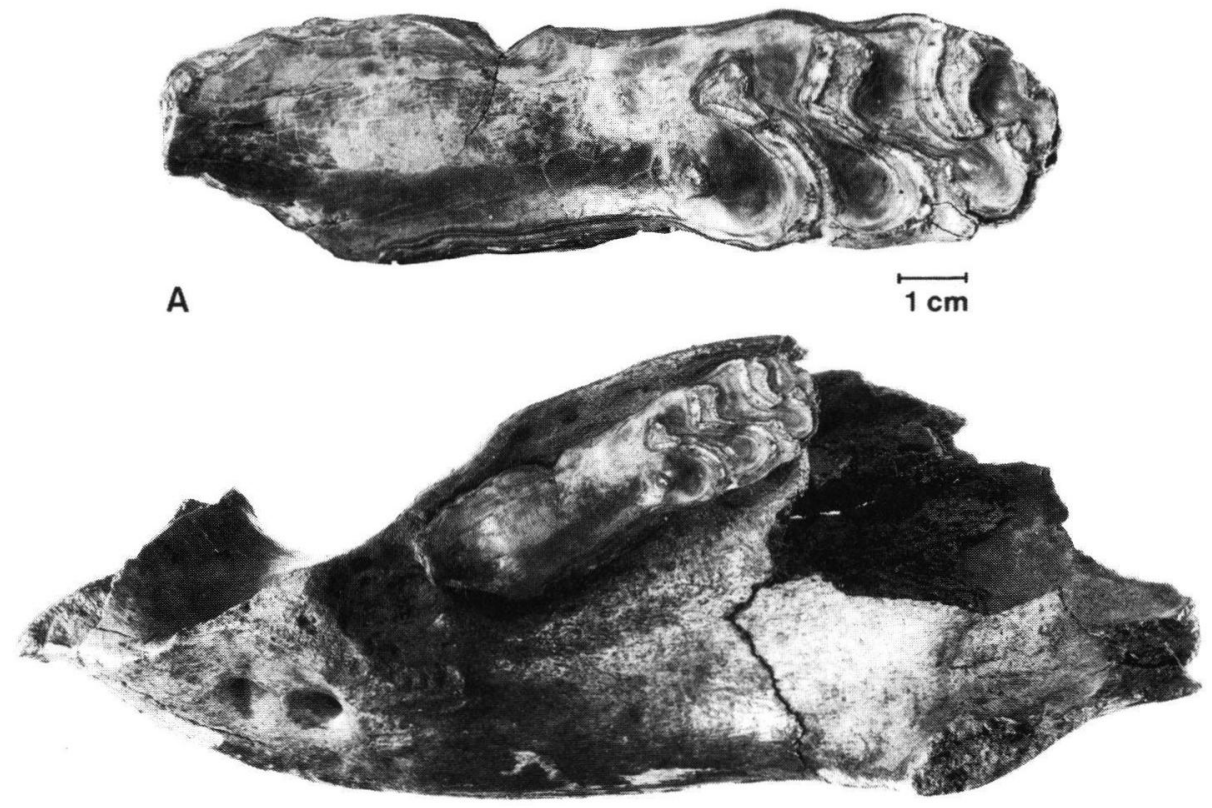

B

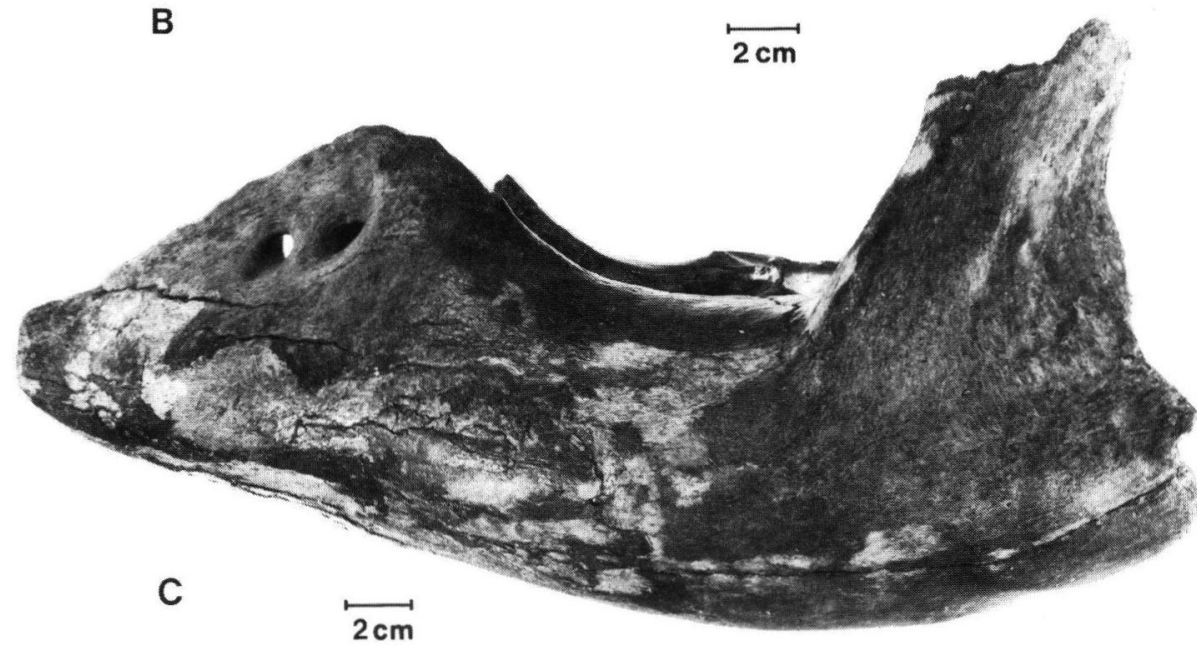

Abb. 8: Mammutbus primigenius. Linke Unterkieferhälfte eines greisenhaften Mammutes aus den jungpleistozänen Rheinschottern bei Nierstein (Hessisches Landesmuseum Darmstadt HLMD-RS 146).

A: Kaufläche des extrem tief abgekauten $\mathrm{M}_{3}$. B: Aufsicht, die die Breite des Kiefers zeigt und C: Seitenansicht des in der Höhe greisenhaft reduzierten Kieferkörpers. 
wahrscheinlich. Allerdings ist die Abkauung der unteren Molaren noch keineswegs soweit vorangeschritten, daß eine weitere Nahrungszerkleinerung für das Tier unmöglich geworden und darin die unmittelbare Todesursache $\mathrm{zu}$ suchen wäre.

Zum Vergleich soll hier ein Unterkieferfragment mit einem noch stärker ausgekauten $\mathrm{M}_{3}$ beschrieben werden (Abb. 8a). Das Stück stammt aus den jungpleistozänen Rheinschottern von Nierstein und wird im Hessischen Landesmuseum Darmstadt (HLMD-RS 146) aufbewahrt. Der noch im Kiefer stehende $M_{3}$ ist auf der ganzen Länge bis in den Wurzelbereich abgekaut und daher nur noch etwa $3 \mathrm{~cm}$ breit. Nur im distalen Teil lassen sich noch die mittleren, besonders tief gelegenen Partien der Schmelzlamellen erkennen. Daß sich ein großer Pflanzenfresser mit derart ausgekauten Zähnen überhaupt noch ernähren konnte, erscheint bemerkenswert. Die Länge des Zahnrudimentes beträgt knapp $14 \mathrm{~cm}$. Der Abkaugrad des dazugehörigen oberen $\mathrm{M}^{3}$ ist leider nicht bekannt. In diesem greisenhaften Alter scheint sich auch bereits der Kieferkörper wesentlich verändert zu haben. Die Breite des Kieferkörpers entspricht der ausgewachsener Mammute, aber seine Höhe ist schon beträchtlich geringer als z. B. beim Mammut von Polch (Abb. 8b und c). Hier ist eine greisenhafte Reduktion der Kieferhöhle wahrscheinlich. Derartige Veränderungen unterliegen ebenfalls starker individueller Variation. Es wäre deswegen lohnend, den Zahnreihen alter Tiere besondere Aufmerksamkeit zu widmen, auch wenn deren Schauwert auf den ersten Blick geringer erscheint.

Bei Mammuten ist es durchaus möglich, eine Vergreisung zu beobachten, da diese Tiere wegen ihrer Körpergröße auch im hohen Alter keine gefährlichen Feinde haben dürften.

\section{Danksagungen}

Ich danke Frau S. WEIERSHÄUSER für ihre Mithilfe bei der Identifizierung der Knochenelemente. Sie hat die Vermessung der Knochen übernommen. Herrn G. OlESCHINSKI danke ich für die Herstellung der Fotos und Frau D. KRaNZ für die Skizzen. Herr Dr. F. SCHRENK, Hessisches Landesmuseum Darmstadt, stellte den Mammutkiefer aus den Rheinschottern freundlicherweise zum Vergleich zur Verfügung.

\section{Schriftenverzeichnis}

ABEL, O. (1929): Rekonstruktion vorzeitlicher Wirbeltiere. 327 S., 254 Abb.; Jena (Fischer).

BosINSKI, G. (1979): Die Ausgrabungen in Gönnersdorf 1968-1976 und die Siedlungsbefunde der Grabung 1968. - Der Magdalénien Fundplatz Gönnersdorf, 3: 1-220. 93 Abb., 20 Taf.; Wiesbaden (F. Steiner).
GUENTHER, E. W. (1955): Mißbildungen an den Backenzähnen diluvialer Elefanten. - Meyniana, 4: 12-36, 2 Abb., 5 Taf.; Hamburg.

Hofer, H. (1939): Polch (Kreis Mayen). - Bonner Jahrbücher, 143/144: 344-349, 1 Abb.; Bonn.

KoENIGSWALD, W. v. (1974): Die pleistozäne Fauna der Weinberghöhlen bei Mauern. - In KOENIGSWALD, W. v., MÜller-Beck, H. \& Pressmar, E.: Archäologie und Paläontologie in den Weinberghöhlen von Mauern. Acta Venatoria, 3: 53-106, 5 Abb., 3 Taf.; Tübingen.

MaUZ, J. (o. J. ? 1936): Mammut-Jagdstelle Polch bei Mayen - eine der bedeutendsten Naturschutzstellen unserer Heimat. - S. 18-21. - In: MAYEN, die Stadt der Burgen und Türme (Veröffentl. der Stadt Mayen).

- (1939): Die Mammut-Jagdstelle bei Polch, Kreis Mayen (Eifel). - Senckenbergiana, 21: 1-26, 34 Abb., 6 Taf.; Frankfurt a. M.

Mordziol, C. (1936): Die Mammutjagdstelle bei Polch. Naturschutz, 17: 254-265, 3 Abb.; Berlin.

- (1939): Einige Beweisstücke für die Deutung des Polcher Mammut-Fundes als Jagdbeute. - Senckenbergiana, 21: 26-37, 32 Abb., 5 Taf.; Frankfurt a. M.

MülleR-Deile, G. (1939): Zu Mauz’ Deutung der ersten Grabung am Polcher Mammut. - Senckenbergiana, 21: 38-41; Frankfurt a. M.

NiedermayeR, J. (1936a): Vor 20000 Jahren lebte das Mammut von Polch. - Generalanzeiger für Bonn und Umgebung vom 25./26. 1. 1936, Bonn.

- (1936b): Auf den Spuren des Urwelttieres. Der Mammutfund bei Polch im Lichte der Wissenschaft. Nationalblatt vom 26. 1. 1936, Koblenz.

OlsEN, S. J. (1972): Osteology for the Archeologist No. 3. The American Mastodon and the wooly Mammoth. Peabody Mus. Archeol. Ethnol. Harvard Univ., 56. 1-45, 36 Abb.; Cambridge Mass.

Roth, V. L. \& Shoshani, J. (1988): Dental identification and age determination in Elephas maximus. - J. Zool., 214: 567-588, 2 Taf., 7 Abb.; London.

SIEGfried, P. (1959): Das Mammut von Ahlen. - Pal. Z., 33: 172-184, 3 Abb., Taf. 19-22; Stuttgart.

- (1983): Fossilien Westfalens: Eiszeitliche Säugetiere. Münster. Forsch. Geol. Paläont., 60: 1-163, 22 Abb., 40 Taf.; Münster.

TOepfer, V. (1957): Die Mammutfunde von Pfännerhall im Geiseltal. - Veröff. Landesmus. Vorgesch. Halle, 16: 1-58, 11 Abb., 24 Taf.; Halle/Saale.

TrevisAN, L. (1948): Lo scheletro di Elephas antiquus italicus di Fonte Campanile (Viterbo). - Palaeontolographia Italica, 44: 1-78, 47 Abb., 5 Taf.; Pisa.

Manuskript eingegangen am 25. 1. 1989. 